\title{
Exogenous Lipoid Pneumonia Following Ingestion of Liquid Paraffin
}

\author{
Akihiko Ohwada, Yasuko Yoshioka, Yuri Shimanuki, Keiko Mitani*, Toshio KumasaKa*, \\ Takashi Dambara and Yoshinosuke FuKUCHI
}

\begin{abstract}
An asymptomatic patient with exogenous lipoid pneumonia (ELP) due to silent aspiration of liquid paraffin ingested as a lubricant was diagnosed by bronchoalveolar lavage (BAL). BAL fluid separated into oily upper phase and lower aqueous phase spontaneously. Microscopic analysis of BAL cells revealed the presence of lipid-laden alveolar macrophages. Classic histochemical staining and electron microscope examination indicated that neutral lipid was dominant but phospholipid was also present in the lipidladen macrophages. Together with the history of ingestion of liquid paraffin, we identified that the ingested liquid paraffin was the origin of the neutral lipid in the lipid-laden macrophages observed in the BAL fluid.

(Internal Medicine 41: 483-486, 2002)
\end{abstract}

Key words: lipid-laden alveolar macrophages, aspiration

\section{Introduction}

Exogenous lipoid pneumonia (ELP) is a rare condition resulting from aspiration or inhalation of oil-based substances (1). Aspiration of liquid paraffin (liquid petrolatum) used for the treatment of constipation or intranasal instillation of liquid paraffin as a component of nasal drop or petroleum jelly such as Vaseline or Mentholatum for relief of sinus congestion is the common cause of ELP (2-4). We herein report an adult patient diagnosed as ELP resulting from aspiration of liquid paraffin used as a lubricant.

\section{Case Report}

A 42-year-old man, non-smoker was referred to our department due to abnormal chest radiographic findings. He works as an architect and denies exposure to chemical substances including paints. Past medical history revealed mechanical ileus 4 years ago, which required surgical correction of the colon invaginated into omental bursa and he suffered from several episodes of recurrent ileus thereafter. He had no fever or respiratory symptoms such as cough, sputum expectoration, or dyspnea. The patient denied obvious aspiration or symptoms of gastroesophageal reflux. Chest auscultation did not reveal any crackles or rhonchi. His chest radiograph revealed pulmonary infiltrate in the right lower lung field adjacent to the right cardiac border (Fig. 1A). Thoracic CT demonstrated a dense alveolar filling in the right middle lobe and linear shadows within it (Fig. 1B). The results of routine laboratory tests were normal. Serum KL-6 was $265 \mathrm{U} / \mathrm{ml}$, with normal value being less than $500 \mathrm{U} / \mathrm{ml}$. Pulmonary function test was normal and no impairment of alveolar diffusion capacity was detected. We performed bronchoalveolar lavage (BAL) and transbronchial lung biopsy of the right middle lobe. The appearance of the BAL fluid was whitish at first and separated spontaneously into an upper oily supernatant layer with visible oil droplets and a lower clear aqueous phase within 5 minutes. The total cell count in the BAL fluid was $1.14 \times 10^{6} / \mathrm{ml}$. The differential count revealed 32 percent macrophages, and 68 percent lymphocytes. The $\mathrm{CD}^{+}$and $\mathrm{CD}^{+}$lymphocytes composed $63.3 \%$ and $22 \%$ of total lymphocytes in the BAL fluid, respectively. The $\mathrm{CD}^{+}{ }^{+}$to $\mathrm{CD}^{+}$ratio was 2.88 . Smear and culture were negative. We evaluated the cytospin preparations of BAL cells with histochemical staining. Nearly all of the alveolar macrophages (AMs) had foamy appearance due to the fact that the cytoplasm was full of numerous, large, rounded vacuoles revealed with the hematoxylin-eosin stain (Fig. 2A). The majority of the vacuoles were stained orange with Oil red O (Fig. 2B). In addition, the vacuoles were stained dark navy blue with heated Sudan black (Fig. 2C), and pink with Nile blue (Fig. 2D). No birefringence was detected in majority of the vacuoles but refringence under polarized light was detected in some of the vacuoles even within the same cells (data not shown). These vacuoles were not stained with the May-Grunwald-Giemsa, Papanicolaou or PAS (data not shown). As for comparison, droplets of liquid paraffin were stained with Oil red O (Fig. 2E), Sudan black (Fig. 2F), and Nile blue (Fig. 2G), respectively. Electron microscope examination revealed that the cytoplasm contained two types of lipid deposits; one was domi-

From the Department of Respiratory Medicine and *the Department of Pathology, Juntendo University School of Medicine, Tokyo

Received for publication November 8, 2001; Accepted for publication February 13, 2002

Reprint requests should be addressed to Dr. Akihiko Ohwada, the Department of Respiratory Medicine, Juntendo University School of Medicine, 2-1-1 Hongo, Bunkyo-ku, Tokyo 113-8421 


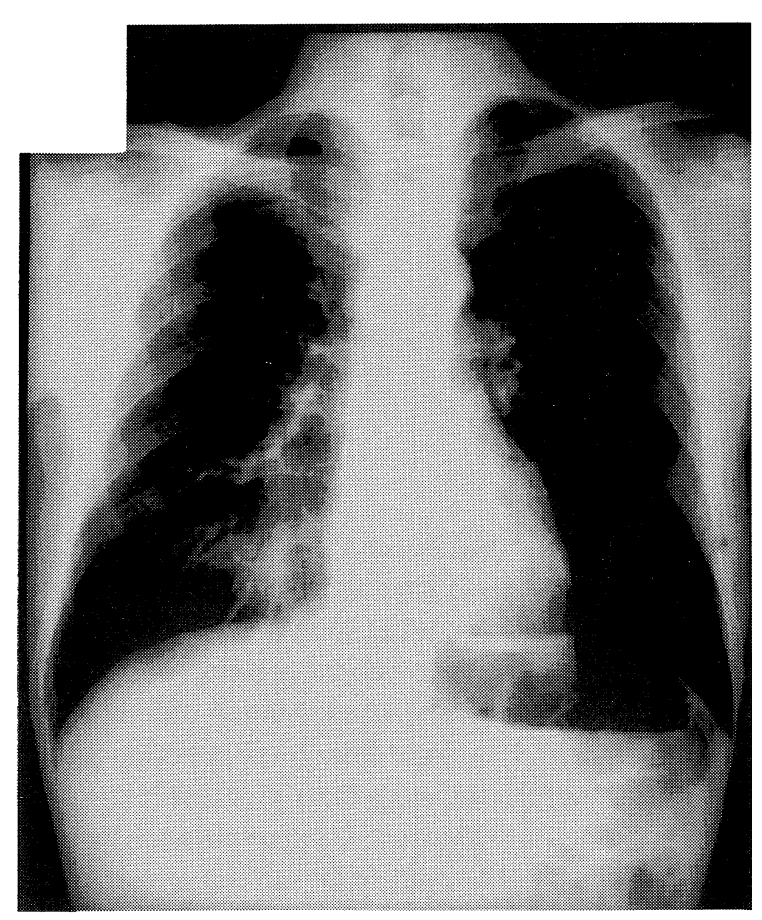

A

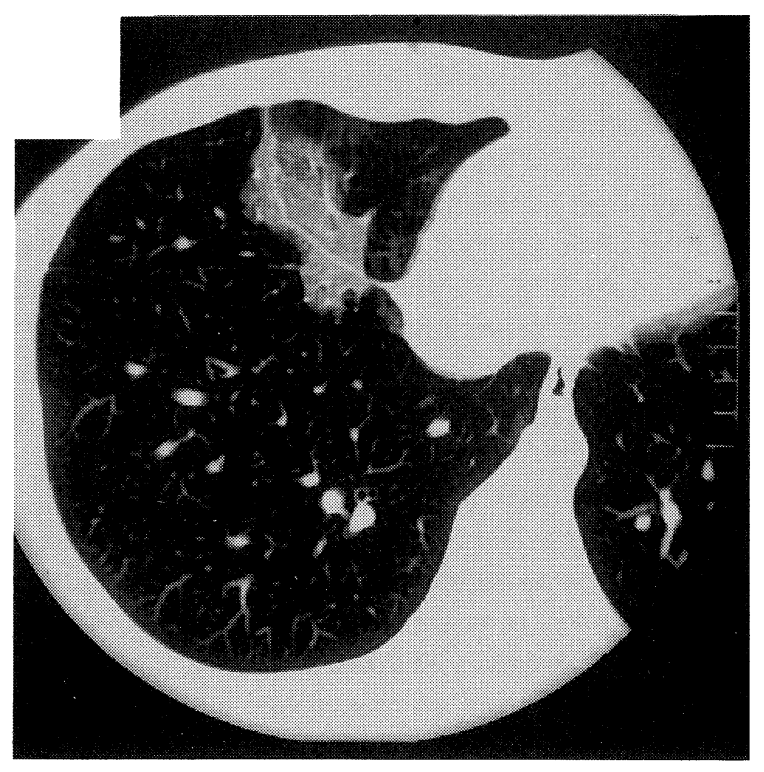

B

Figure 1. A: Chest radiograph revealing pulmonary infiltrate in the right lower lung field. B: Thoracic CT scan revealed dense alveolar infiltration in the right middle lobe.

nantly moderate osminophilic lipid or vacuoles that coincided with majority of the vacuoles in the AMs observed by the light microscope (Fig. 3A, B), the other was multilamellated surfactant (Fig. 3B). Lipid analysis of the BAL fluid revealed that the fluid contained $0.03 \mathrm{mg} / \mathrm{ml}$ phospholipid that was com- posed of $53.4 \%$ phosphatidyl serine and phosphatidyl inositol and $41.2 \%$ phosphatidyl choline. But triglyceride and free fatty acid were not detected in the BAL fluid. ELP was diagnosed on the basis of macroscopic evaluation of BAL fluid and microscopic analyses of cytospin preparation of BAL fluid. Past history revealed that he had been ingesting liquid paraffin daily as a lubricant for two and a half years. The daily dose of liquid paraffin was initially commenced at $30 \mathrm{ml}$ but gradually increased to $60 \mathrm{ml}$ and maintained at this dose for the last 14 months. These past 2 months, the daily dose of liquid paraffin was increased to $90 \mathrm{ml}$. Together with the history of ingestion of liquid paraffin it was evident that liquid paraffin was the major source of exogenous lipid in this case. Histochemical staining of droplets of liquid paraffin was consistent with the findings of the staining of lipids inside the AMs. Result of transbronchial lung biopsy revealed intra-alveolar accumulation of lipid-laden macrophages and interstitial pneumonitis composed of lymphocytic infiltration, deposits of fat droplets and fibrotic proliferation within the alveolar septa (Fig. $2 \mathrm{H}$ ).

\section{Discussion}

In this particular case, the macroscopic evaluation of the BAL fluid that separated into upper oily supernatant layer and a lower clear aqueous layer strongly suggests the diagnosis of ELP. Histological examination revealed the presence of lipidladen macrophages, and histochemical lipid staining was useful to differentiate the type of lipids phagocytosed by this patient's AMs $(1,5)$. The results revealed the presence of two types of lipids in the AMs; one was a dominant neutral lipid and the other was a phospholipid. Electron microscope examination confirmed these findings by demonstrating osminophilic lipid and surfactant rich in phospholipid such as phosphatydil choline (PC). The value of phospholipid in BAL fluid was within the normal range (6). The lipid analysis of BAL fluid revealed a decrease of phosphatidyl choline (PC) and an increase of phosphatidyl inositol/phosphatidyl serine similar to the findings observed in patients with pulmonary alveolar proteinosis (6). Thoracic CT of this patient revealed a patchy well-defined area of ground-glass attenuation with superimposed septal thickening (crazy-paving pattern) (see Fig. 1B). Franquet et al indicated that this finding is one of the characteristics observed in patients with ELP (7).

There are many oil-based substances that cause ELP such as vegetable oil (olive), animal oil (milk, cod-liver oil) and mineral oil (1). However, mineral oil such as liquid paraffin is one of the most frequent sources of exogenous lipid (1). It is postulated that mineral oils could inhibit the cough reflex and ciliary motility, thus facilitating inhalation (8). Another study revealed that mineral oil applied on rabbit and human nasal epithelium markedly impaired the movement of the mucus blanket by altering the physical properties of the secretions (9). These characteristics of mineral oil could fasciculate silent aspiration of liquid paraffin as presented in this case. Although infrared spectrophotometry would confirm the presence of liquid paraffin in AMs (10), it is restricted to special laboratories 

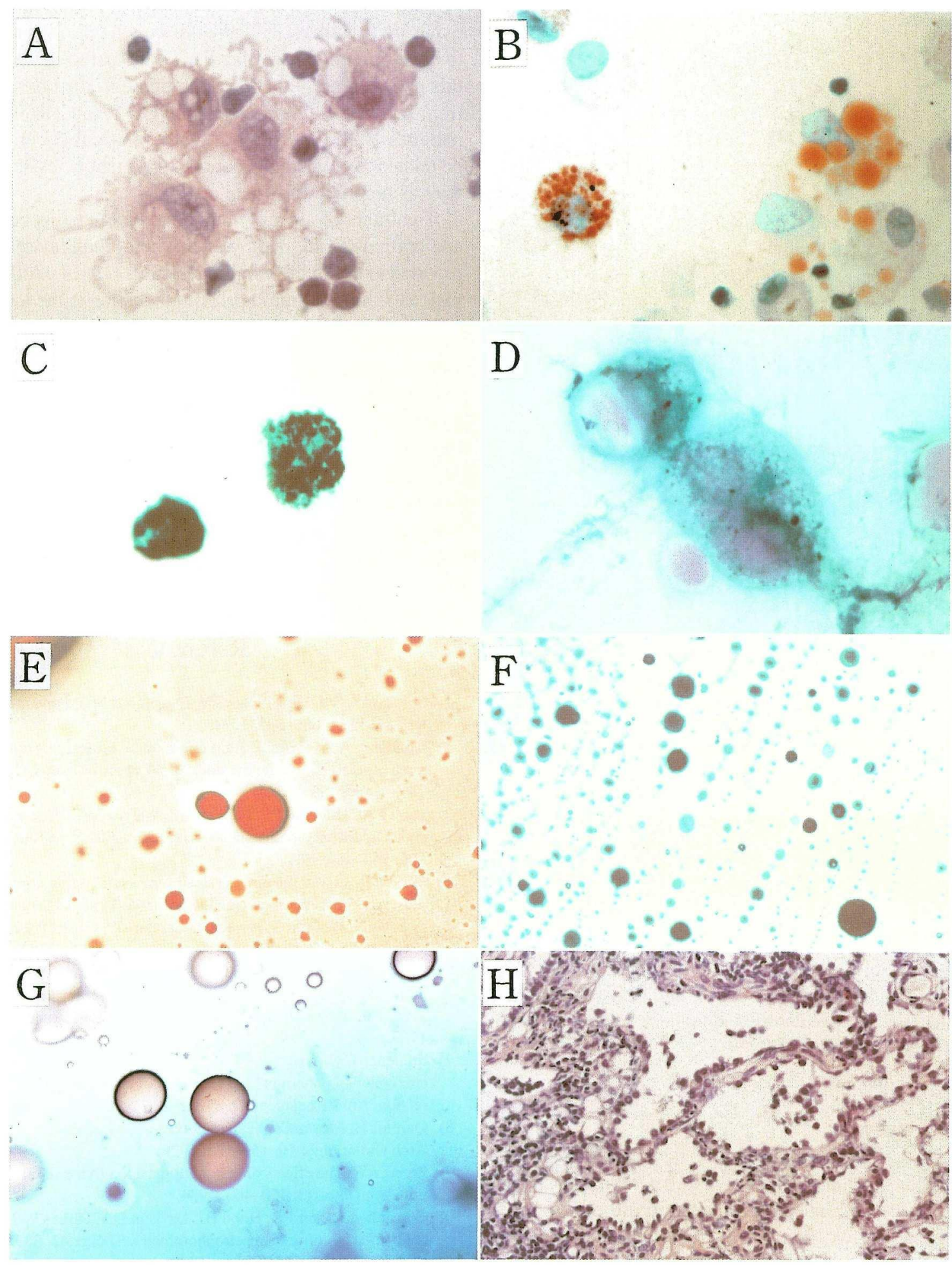

Figure 2. Histological examination of the alveolar macrophages (AMs) obtained from this patient with ELP and droplets of liquid paraffin. A: BAL cells were stained with HE stain $(\times 300)$. Note the vacuoles in the AMs and the activation of the AMs with the spreading pod-like processes. The lymphocytes were also identified around the AMs. B: AM stained orange with Oil red O ( $\times 250)$. C: AM stained dark navy blue with Sudan black $(\times 250)$. D: AM were stained pink with Nile blue $(\times 300)$. E: Droplet of liquid paraffin stained with Oil red $O(\times 4)$. F: Droplet of liquid paraffin stained with Sudan black $(\times 4)$. G: Droplet of liquid paraffin stained with Nile blue $(\times 4)$. H: The transbronchial lung biopsy demonstrating interstitial pneumonitis with infiltrated lymphocytes and deposit of fat droplets $(\times 160)$. 


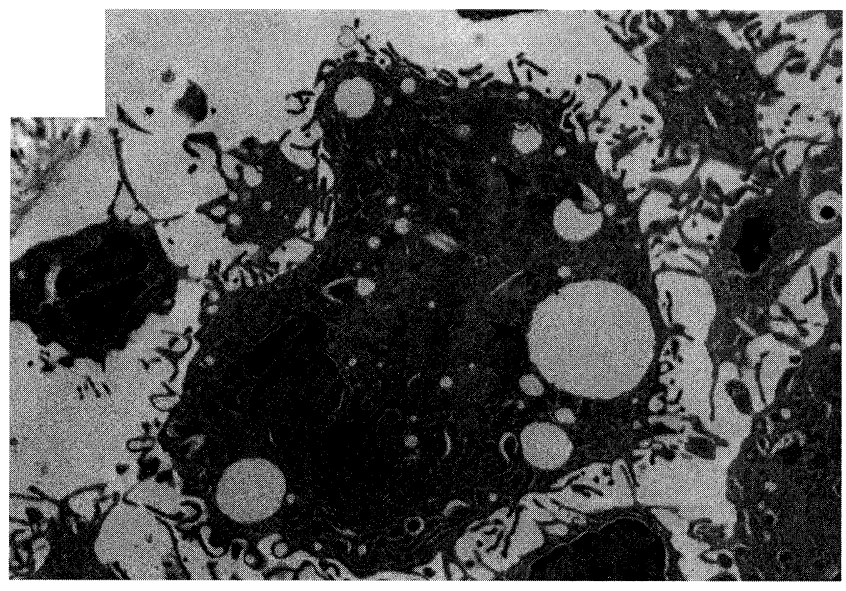

A

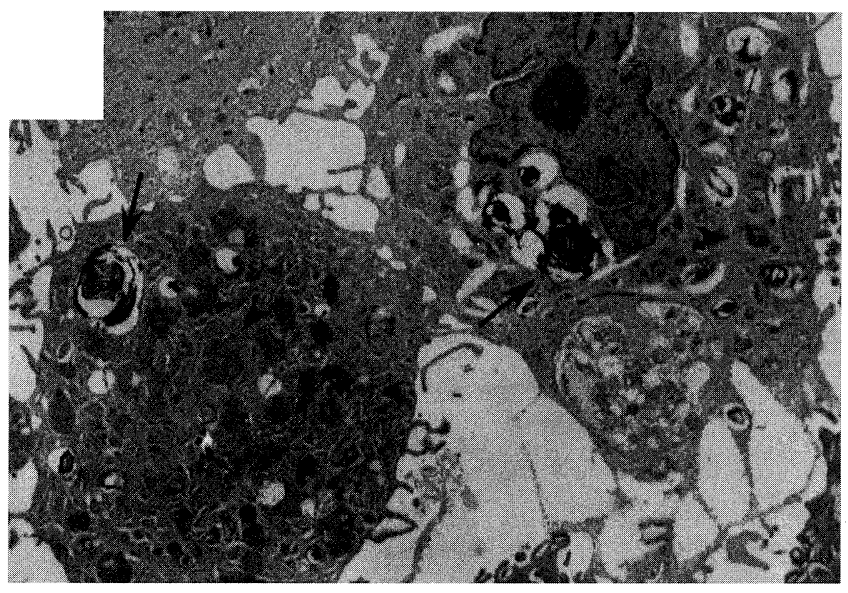

$\mathrm{B}$

Figure 3. Electron micrograph of alveolar macrophages (AMs) obtained by BAL from this case. Note two types of lipid deposits in the alveolar macrophages in $A(\times 1,830)$ and $B(\times 1,650)$. Osmiophilic lipid or vacuoles were dominantly distributed (arrowheads) in the AMs. Multilamellated surfactants phagocytosed by AMs were also identified (arrows).

and it is very expensive.

Post mineral oil ingestion, the initial response in the alveoli is the phagocytosis of emulsified oil by alveolar macrophages. These lipid-laden macrophages are activated to release proinflammatory cytokines to elicit foreign body reaction associated with the infiltration of lymphocytes and plasma cells into the alveolar septum $(1,11)$. With time, interstitial fibrosis develops, and in some cases large oil droplets walled by fibrous tissues and giant cells creating a tumor-like mass called paraffinoma forms $(1,2)$. Electron microscopic examination revealed that the majority of the AMs obtained from the present case had pod-like processes suggesting "activated" macrophages (see Figs. 2A and 3). Pulmonary biopsy revealed the presence of interstitial pneumonitis coinciding with the shadow of lipoid pneumonia observed in the chest radiograph. Although exposure to mineral oil for a prolonged period is not required to develop pulmonary fibrosis (1), the pathological finding of this patient represents the initial phase of the disease. In addition, increased lymphocytes and $\mathrm{CD} 4^{+}$to $\mathrm{CD} 8^{+}$ratio in this patient's BAL fluid suggest that interstitial pneumonitis may be related to cooperative actions of lymphocytes and macrophages as reported elsewhere (12).

Treatment of asymptomatic patients remains controversial and corticosteroid therapy and (whole) lung lavage have been reported to be effective in limited cases $(13,14)$. Although complete cessation of liquid paraffin ingestion is the ideal primary treatment but it was difficult to abruptly stop it completely due to the patient's mental dependency to liquid paraffin in this case. Hence, the patient was instructed to decrease the dose of liquid paraffin to $30 \mathrm{ml}$ per day, since the chest radiographic examination of a year earlier appeared to be normal with $30 \mathrm{ml}$ ingestion of liquid paraffin per day. Regular follow-up chest radiographic examinations are scheduled to determine whether the shadow is attenuated at this dose of liquid paraffin. Therefore, it is worthy of mention that a comprehensive medical history, especially medication and the habits of the patient is important and essential as demonstrated in this particular case.

\section{References}

1) Spickard A 3rd, Hirschmann JV. Exogenous lipoid pneumonia. Arch Intern Med 154: 686-692, 1994.

2) Gondouin A, Manzoni P, Ranfaing E, et al. Exogenous lipid pneumonia: a retrospective multicentre study of 44 cases in France. Eur Respir J 9: 1463-1469, 1996.

3) Brown AC, Slocum PC, Putthoff SL, Wallace WE, Foresman BH. Exogenous lipoid pneumonia due to nasal application of petroleum jelly. Chest 105: 968-969, 1994.

4) Varkey B. Lipoid pneumonia due to intranasal application of petroleum jelly. An old problem revisited. Chest 106: 1311-1312, 1994.

5) Lauque D, Dongay G, Levade T, Caratero C, Carles P. Bronchoalveolar lavage in liquid paraffin pneumonitis. Chest 98: 1149-1155, 1990.

6) Honda Y, Kataoka K, Hayashi H, Takahashi H, Suzuki A, Akino T. Alterations of acidic phospholipids in bronchoalveolar lavage fluids of patients with pulmonary alveolar proteinosis. Clin Chim Acta 181: 11-18, 1989.

7) Franquet T, Gimenez A, Bordes R, Rodriguez-Arias JM, Castella J. The crazy-paving pattern in exogenous lipoid pneumonia: CT-pathologic correction. Am J Roentgenol 170: 315-317, 1998.

8) Quinn LH, Meyer OO. The relationship of sinusitis and bronchiectasis. Arch Otolaryngol 10: 152-165, 1929.

9) Proetz AW. The effects of certain drugs upon living nasal ciliated epithelium. Ann Otol 43: 450-463, 1934.

10) Corrin B, Crocker PR, Hood BJ, Levison DA, Parkes WR. Paraffinoma confirmed by infrared spectrophotometry. Thorax 42: 389-390, 1987.

11) Midulla F, Strappini PM, Ascoli V, et al. Bronchoalveolar lavage cell analysis in a child with chronic lipid pneumonia. Eur Respir J 11: 239242, 1998.

12) Russi TJ, Crystal RG. Use of Bronchoalveolar Lavage and Airway Brushing to Investigate the Human Lung. in: The Lung: Scientific Foundations. Crystal RG Ed. Lippincott-Raven Publishers, Philadelphia, 1997: 373-374.

13) Chin NK, Hui KP, Sinniah R, Chan TB. Idiopathic lipoid pneumonia in an adult treated with prednisolone. Chest 105: 956-957, 1994.

14) Chang HY, Chen CW, Chen CY, et al. Successful treatment of diffuse lipoid pneumonitis with whole lung lavage. Thorax 48: 947-948, 1993. 